\title{
Unlike pregnant adult women, pregnant adolescent girls cannot maintain glycine flux during late pregnancy because of decreased synthesis from serine
}

\author{
Jean W. Hsu ${ }^{1}$, Minerva M. Thame ${ }^{2}$, Raquel Gibson ${ }^{3}$, Tameka M. Baker ${ }^{2}$, Grace J. Tang ${ }^{1}$, Shaji K. Chacko ${ }^{1}$, \\ Alan A. Jackson ${ }^{4}$ and Farook Jahoor ${ }^{1 *}$ \\ ${ }^{1}$ US Department of Agriculture/Agricultural Research Service, Department of Paediatrics, Children's Nutrition Research Center, \\ Baylor College of Medicine, Houston, TX 77030, USA \\ ${ }^{2}$ Department of Child and Adolescent Health, University of the West Indies, Mona, Kingston-7, Jamaica \\ ${ }^{3}$ Department of Obstetrics and Gynaecology, University of the West Indies, Mona, Kingston-7, Jamaica \\ ${ }^{4}$ National Institute for Health Research (NIHR) Southampton Biomedical Research Centre, Southampton General Hospital \\ (MP 113), Tremona Road, Southampton SO16 6YD, UK
}

(Submitted 3 September 2015 - Final revision received 27 October 2015 - Accepted 7 December 2015)

\section{Abstract}

During pregnancy, glycine and serine become more important because they are the primary suppliers of methyl groups for the synthesis of fetal DNA, and more glycine is required for fetal collagen synthesis as pregnancy progresses. In an earlier study, we reported that glycine flux decreased by $39 \%$ from the first to the third trimester in pregnant adolescent girls. As serine is a primary precursor for glycine synthesis, the objective of this study was to measure and compare glycine and serine fluxes and inter-conversions in pregnant adolescent girls and adult women in the first and third trimesters. Measurements were made after an overnight fast by continuous intravenous infusions of ${ }^{2} \mathrm{H}_{2}$-glycine and

${ }^{15} \mathrm{~N}$-serine in eleven adolescent girls (17.4 (sE 0.1) years of age) and in ten adult women (25.8 (SE 0.5) years of age) for $4 \mathrm{~h}$. Adolescent girls had significantly slower glycine flux and they made less glycine from serine in the third $(P<0.05)$ than in the first trimester. Baby birth length was significantly shorter of adolescent girls $(P=0.04)$ and was significantly associated with third trimester glycine flux. These findings suggest that the pregnant adolescent cannot maintain glycine flux in late pregnancy compared with early pregnancy because of decreased synthesis from serine. It is possible that the inability to maintain glycine synthesis makes her fetus vulnerable to impaired cartilage synthesis, and thus linear growth.

Key words: Pregnant adolescent girls: Pregnant adult women: Glycine: Serine

Pregnancy during adolescence in Jamaica and elsewhere is associated with a high prevalence of low birth weight ${ }^{(1,2)}$. It has been proposed that this is due to an inability of the adolescent mother to provide the nutrient needs for her own growth and the growth of her fetus. In particular, the requirement for amino acids increases as pregnancy progresses in order to sustain increased rates of protein deposition ${ }^{(3)}$ and to support increased availability of glucose through gluconeogenesis ${ }^{(4)}$.

Dispensable amino acids comprise the bulk of maternal amino acids transferred to the fetus in pregnancy ${ }^{(5)}$. A good example is glycine, which is a provider of methyl groups needed for the synthesis of DNA necessary for cell division to support maternal and fetal tissue deposition ${ }^{(6,7)}$. In addition, most of fetal cartilage is synthesised during late pregnancy and glycine constitutes $25 \%$ of the amino acids in cartilage ${ }^{(8)}$. Therefore, as pregnancy progresses, there is a higher fetal demand for glycine. From our earlier study, glycine flux decreased by $39 \%$ from the first trimester to the third trimester in pregnant adolescent girls ${ }^{(9)}$. Plasma glycine concentration in pregnant adolescent girls also decreased significantly from trimester 1 to 3, indicating a reduced availability. These findings suggested that after an overnight fast pregnant adolescent girls had a shortage in glycine supply in late pregnancy because they could not maintain production similar to their adult counterparts. However, de novo synthesis of glycine during pregnancy is not known. On the basis of evidence that most of de novo glycine synthesis is from serine in humans ${ }^{(10)}$, we hypothesised that unlike adult counterparts adolescent girls cannot maintain glycine flux in late pregnancy because of decreased synthesis from serine. The objective of the present study was to measure and compare glycine and serine kinetics of pregnant adolescent girls and adult women in the first and third trimesters. The present study is part of the phase 2 study of a larger study of amino acid metabolism in pregnancy ${ }^{(11)}$.

\section{Methods}

This study was conducted according to the guidelines laid down in the Declaration of Helsinki, and all the procedures involving human subjects were approved by the Ethics Committee of the University of the West Indies and by the Institutional Review 
Board for Human Subject Research of Baylor College of Medicine \& Affiliated Hospitals. Written informed consent was obtained from each study participant at recruitment.

In total, eleven pregnant adolescent girls, aged 16-17 years, and twelve pregnant adult women attending the antenatal clinic at the University Hospital of the West Indies were invited to participate in the study, and were enrolled consecutively. Women with chronic illnesses such as diabetes mellitus, hypertension, heart disease or a genetic abnormality such as sickle cell disease or women with multiple gestations were excluded. All the adolescents were studied at 13 and 29 weeks of gestation. The twelve adult women were studied at 13 weeks of gestation; however, only ten adults were studied at 29 weeks of gestation because one had a spontaneous abortion and the other had a premature delivery at 24 weeks and the baby did not survive. Data from these two adults were excluded from the analysis.

Maternal weight was measured at the time of each study to the nearest $0.01 \mathrm{~kg}$ using a Tanita digital scale (CMS Weighing Equipment Ltd). Maternal weight gain from the first to the third trimester was calculated. Maternal height was measured at the first study to the nearest $0 \cdot 1 \mathrm{~cm}$ using a stadiometer (CMS Weighing Equipment Ltd). Gestational age was determined by the last menstrual period and confirmed by an ultrasound measurement performed at the time of the first experimental study. Birth weight was measured to the nearest $0 \cdot 01 \mathrm{~kg}$ using a Tanita model 1583 digital baby scale (CMS Weighing Equipment Ltd); crown-heel length was measured to the nearest $0 \cdot 1 \mathrm{~cm}$ using a Harpenden infantometer (CMS Weighing Equipment Ltd); and head circumference was measured using a fibre-glass tape measure.

\section{Tracer infusion protocol}

All the participants were studied after an 8-h overnight fast on two occasions: $13 \cdot 1$ (sE $0 \cdot 4$ ) weeks of gestation (trimester 1 ) and 28.8 (sE 0.4) weeks of gestation (trimester 3). Participants were admitted to the obstetric ward in the evening and were allowed to have their last meal of the day at 22.00 hours. After $8 \mathrm{~h}$, an intravenous catheter (Sensecure, 18G; Morningside Pharmaceuticals Ltd) was inserted into the antecubital vein of one arm for the infusion of isotopes, whereas a second catheter was inserted in an anti-flow direction into the dorsal vein of the contralateral hand for drawing blood samples. The cannula was kept patent with intermittent infusions of heparinised saline.

Sterile solutions of $2,2-{ }^{2} \mathrm{H}_{2}$-glycine and ${ }^{15} \mathrm{~N}$-serine (Cambridge Isotope Laboratories) were prepared in isotonic saline. Baseline blood samples were collected before the start of the infusion protocol. Simultaneous primed constant infusions of ${ }^{2} \mathrm{H}_{2}$-glycine and ${ }^{15} \mathrm{~N}$-serine (prime $=4 \mu \mathrm{mol} / \mathrm{kg}$, infusion $=4 \mu \mathrm{mol} / \mathrm{kg}$ per $\mathrm{h}$, respectively) were initiated and maintained for $4 \mathrm{~h}$. Further blood samples were collected at 10-min intervals during the last 30 min of the infusions. At the end of each of the tracer infusion periods, the catheters were removed, the subjects were provided lunch and discharged.

\section{Laboratory analyses}

Blood samples were drawn into pre-chilled tubes containing heparin, centrifuged at $4^{\circ} \mathrm{C}$ and the plasma was separated and stored at $-70^{\circ} \mathrm{C}$ for later analysis.
The plasma glycine and serine isotopic enrichments were measured by liquid chromatography-tandem MS. In brief, plasma glycine and serine were converted into their 5-(dimethylamino)-1napthalenesulphonamide derivatives and analysed using a Kinetex C18 $2 \cdot 6 \mu 100 \times 2 \cdot 1 \mathrm{~mm}$ column (Phenomenex) on a triple quadrupole mass spectrometer (TSQ Vantage; Thermo Scientific), equipped with an heated-electrospray ionisation source, a Accela pump (Thermo Scientific) and a Thermal PAL autosampler (Thermo Scientific). The ions were then analysed in the selected reaction monitoring mode. The transitions observed were precursor ions $\mathrm{m} / \mathrm{z} 309,310$ and 311 to product ion $\mathrm{m} / z 170$ at $19 \mathrm{eV}$ for glycine and precursor ion $\mathrm{m} / z 339,340$ to product ion $\mathrm{m} / z 170$ at $20 \mathrm{eV}$ for serine. Instrumental control, data acquisition and analysis were performed by Xcalibur (version 2.1) software package (Thermo Scientific).

The ${ }^{2} \mathrm{H}$ enrichment of plasma serine derived from the conversion of ${ }^{2} \mathrm{H}_{2}$-glycine was measured after the separation and subsequent pyrolysis utilising GC-thermal conversion-isotope ratio MS technique (GC-TC-IRMS; Thermo Scientific Delta ${ }^{+} \mathrm{XL}$ ). In brief, the amino acids in the plasma samples were first separated by Dowex AG-50W resin columns (Bio-Rad Life Sciences Research) and esterified using propanol-HCl (3:1) by incubating at $70^{\circ} \mathrm{C}$ for $1 \mathrm{~h}$. Subsequently, the esterified samples were acetylated by acetone-triethylamine-acetic anhydride (5:2:2) and incubated at $70^{\circ} \mathrm{C}$ for $20 \mathrm{~min}$. The samples were then injected into the GC-TC-IRMS for separation followed by pyrolysis to $\mathrm{H}$ in order to determine ${ }^{2} \mathrm{H}$ enrichment of plasma serine.

\section{Calculations}

Total glycine or serine flux $(Q)$ was calculated as follows:

$$
Q(\mu \mathrm{mol} / \mathrm{kg} \text { per h })=I \times\left(I E_{\mathrm{inf}} / I E_{\mathrm{pl}}\right),
$$

where $I E_{\text {inf }}$ is the isotopic enrichment of the infusate, $I E_{\mathrm{pl}}$ is the plateau isotopic enrichment of either glycine or serine in plasma and $I$ is the tracer infusion rate. Enrichment of ${ }^{15} \mathrm{~N}$-serine was obtained as the difference between the measured $m+1$ serine enrichment and the enrichment of ${ }^{2} \mathrm{H}$-serine derived from the ${ }^{2} \mathrm{H}_{2}$-glycine tracer.

Endogenous glycine or serine flux was obtained by subtracting the tracer infusion rate.

The rate of conversion of serine to glycine $\left(Q_{\mathrm{Ser} \rightarrow \mathrm{Gly}}\right)$ was calculated as follows:

$$
Q_{\mathrm{Ser} \rightarrow \mathrm{Gly}}=Q_{\mathrm{Gly}} \times \mathrm{IE}_{\mathrm{Gly}} / \mathrm{IE}_{\mathrm{Ser}},
$$

where $Q_{\mathrm{Gly}}$ is the total glycine flux, $I E_{\mathrm{Gly}}$ is the plateau ${ }^{15} \mathrm{~N}$-glycine enrichment in plasma and $I E_{\text {Ser }}$ is the plateau ${ }^{15} \mathrm{~N}$-serine enrichment in plasma.

The rate of conversion of glycine to serine $\left(Q_{\mathrm{Gly}} \rightarrow \mathrm{Ser}\right)$ was calculated as follows:

$$
Q_{\mathrm{Gly} \rightarrow \mathrm{Ser}}=Q_{\mathrm{Ser}} \times \mathrm{IE}_{\mathrm{Ser}} / \mathrm{IE}_{\mathrm{Gly}}
$$

where $Q_{\text {Ser }}$ is the total serine flux, $I E_{\text {Ser }}$ is the plateau ${ }^{2} \mathrm{H}$-serine enrichment in plasma and $I E_{\mathrm{Gly}}$ is the plateau ${ }^{2} \mathrm{H}_{2}$-glycine enrichment in plasma. 


\section{Statistical analysis}

Data are expressed as mean values with their standard errors. Maternal characteristics and pregnancy outcomes of the two groups were assessed using the non-paired $t$ test. The D'Agostino-Pearson test was used to check for normalcy of distribution of the study data. The Grubb's method was used to remove outliers. Differences in amino acid variables between the groups were analysed by mixed-model (repeated-measure two-factor) ANOVA. This model included the two age groups (adult and adolescent) and time of pregnancy (first and third trimesters). Post hoc comparisons were performed using Sidak's test. As each group had different body weights, total body glycine and serine fluxes were not compared among groups. Only within-group comparisons were made for trimester 1-3 using the paired $t$ test. Tests were considered statistically significant if $P<0.05$. Pearson's correlation (one-tailed) was performed on newborn length and maternal third trimester glycine flux. Data analyses were performed using GraphPad Prism version 6 software (GraphPad Software).

\section{Results}

The maternal characteristics and pregnancy outcomes of the subjects have been published previously ${ }^{(11)}$. However, for convenience, some of the relevant data are presented in Table 1. In brief, at 13 weeks of gestation, there were no significant differences in body weight or BMI between the groups, although the adolescents tended to be lighter with lower BMI compared with the adults. Similarly, there were no significant differences in body weight at 29 weeks of gestation and in weekly weight gain from week 13 to 29 of gestation. Gestational age at birth was significantly longer in the adolescent group (39.5 (sE 0.3) weeks) compared with the adult group (38 (SE 0.4 ) weeks; $P<0.05$ ), but the average length of their newborn babies was significantly shorter, with the crown-heel length being $47 \mathrm{~cm}$ compared with $49.5 \mathrm{~cm}(P<0 \cdot 05)$.

When glycine kinetic parameters were expressed per kilogram of body weight, there was a significant effect of trimester on endogenous glycine flux (Table 2). Endogenous glycine flux and glycine flux derived from serine were significantly slower in adolescent girls in trimester 3 than in trimester $1(P<0 \cdot 05)$.
There was a significant interaction between age group and time of pregnancy $(P<0.05)$ on glycine flux derived from serine (Fig. 1), as glycine flux derived from serine decreased significantly from trimester 1 to 3 in the adolescent group but not in the adult group. When the kinetic parameters were expressed per whole body, endogenous glycine flux and glycine flux derived from serine were not different from trimester 1 to 3 in both groups.

There was no significant effect of trimester or age group on endogenous serine flux and serine flux derived from glycine, when the kinetic parameters were expressed either per kilogram of body weight or per whole body (Table 2 ).

There was a positive association between newborn crownheel length and endogenous glycine flux in trimester 3 in adolescent girls $(P=0.05)$ but not in adult women (Fig. 2$)$.

\section{Discussion}

To test the hypothesis that adolescent girls cannot maintain glycine flux in late pregnancy because of decreased synthesis from serine, endogenous glycine and serine fluxes, glycine flux derived from serine and serine flux derived from glycine were measured in adult women and adolescent girls after an overnight fast at the end of the first trimester and at the beginning of the third trimester of pregnancy. The results show that in pregnant adolescents glycine and serine fluxes and conversion of serine to glycine were significantly slower at trimester 3 compared with trimester 1 . These findings corroborate our earlier finding that glycine flux decreased in the adolescent group in trimester 3, suggesting that the pregnant adolescent cannot maintain glycine production in late pregnancy because of decreased de novo synthesis from serine ${ }^{(9)}$.

The findings of the present study have several important implications for fetal development. Glycine has several critical functions with respect to the development of the fetus, which includes being a primary source of one-carbon necessary for both synthesis and methylation of DNA and new proteins ${ }^{(7)}$. It is also in high demand to synthesise glycine-rich collagen in late gestation $^{(8)}$. Therefore, its availability is critical for optimal fetal growth. Our results showed that in adolescent mothers glycine flux in trimester 3 had a positive association with the baby's

Table 1. Maternal characteristics and pregnancy outcomes of study subjects (Mean values with their standard errors)

\begin{tabular}{|c|c|c|c|c|}
\hline \multirow[b]{2}{*}{ Variables } & \multicolumn{2}{|c|}{ Adults $(n 10)$} & \multicolumn{2}{|c|}{ Adolescents ( $n$ 11) } \\
\hline & Mean & SE & Mean & SE \\
\hline \multicolumn{5}{|l|}{ Maternal characteristics } \\
\hline Age (years)* & $25 \cdot 8$ & 0.5 & $17 \cdot 4$ & 0.1 \\
\hline Weight at 13th week of gestation $(\mathrm{kg})$ & 58.4 & $1 \cdot 7$ & 55.5 & $2 \cdot 3$ \\
\hline BMI $\left(\mathrm{kg} / \mathrm{m}^{2}\right)$ & 22 & 0.6 & 21 & 0.8 \\
\hline Weight at 29th week of gestation $(\mathrm{kg})$ & $64 \cdot 2$ & 1.6 & 62.5 & 2.5 \\
\hline Weight gain from 13th to 29th week of gestation ( $\mathrm{kg} / \mathrm{week}$ ) & 0.35 & 0.07 & 0.45 & 0.07 \\
\hline \multicolumn{5}{|l|}{ Pregnancy outcomes } \\
\hline Gestational age at birth (weeks) ${ }^{*}$ & $38 \cdot 0$ & 0.4 & 39.5 & 0.3 \\
\hline Birth weight $(\mathrm{kg})$ & 3.35 & 0.13 & $3 \cdot 11$ & 0.1 \\
\hline Crown-heel length $(\mathrm{cm})^{\star}$ & 49.5 & 0.6 & $47 \cdot 0$ & 0.9 \\
\hline
\end{tabular}

* Significantly different: $P<0.05$ (unpaired $t$ test). 
Table 2. Glycine and serine kinetics in pregnant adolescent girls and adult women at 13 and 29 weeks of gestation (Mean values with their standard errors)

\begin{tabular}{|c|c|c|c|c|c|c|c|c|}
\hline \multirow[b]{3}{*}{ Kinetics } & \multicolumn{4}{|c|}{ Adults $(n 10)$} & \multicolumn{4}{|c|}{ Adolescents ( $n$ 11) } \\
\hline & \multicolumn{2}{|c|}{ Trimester $1^{*}$} & \multicolumn{2}{|c|}{ Trimester $3^{*}$} & \multicolumn{2}{|c|}{ Trimester $1^{*}$} & \multicolumn{2}{|c|}{ Trimester $3^{*}$} \\
\hline & Mean & SE & Mean & SE & Mean & SE & Mean & SE \\
\hline \multicolumn{9}{|c|}{ Per kg body weight ( $\mu \mathrm{mol} / \mathrm{kg}$ per $\mathrm{h})$} \\
\hline Gly endogenous flux & 141 & 11.4 & 125 & 3.9 & 165 & $10 \cdot 7$ & $142 \ddagger$ & $6 \cdot 9$ \\
\hline Gly flux derived from Ser§ & 51.6 & $5 \cdot 1$ & $56 \cdot 1$ & 8.5 & 69.9 & $6 \cdot 8$ & $54.0 \ddagger$ & 3.4 \\
\hline Ser endogenous flux & 112 & $10 \cdot 0$ & 105 & $7 \cdot 3$ & 134 & $10 \cdot 7$ & 111 & $5 \cdot 5$ \\
\hline Ser flux derived from Gly & 4.19 & 0.60 & $4 \cdot 16$ & 0.53 & $4 \cdot 28$ & 0.62 & $5 \cdot 11$ & 0.43 \\
\hline \multicolumn{9}{|l|}{ Per whole body $(\mu \mathrm{mol} / \mathrm{h})$} \\
\hline Gly endogenous flux & 8342 & 961 & 8129 & 398 & 9047 & 576 & 8817 & 416 \\
\hline Gly flux derived from Ser & 3627 & 644 & 3680 & 563 & 3827 & 361 & 3328 & 172 \\
\hline Ser endogenous flux & 7603 & 1073 & 7743 & 1051 & 7347 & 616 & 6881 & 305 \\
\hline Ser flux derived from Gly & 249 & 37.3 & 269 & 37.3 & 222 & 29.9 & 298 & $21 \cdot 1$ \\
\hline
\end{tabular}

* Trimester $1=13.1$ (SE 0.4) weeks of gestation; trimester $3=28.8$ (SE 0.4) weeks of gestation.

† Significant effect of trimester: $P<0.05$ (repeated-measures two-factor ANOVA).

$\ddagger$ Significantly different from corresponding trimester 1 value: $P<0.05$ (post hoc Sidak's multiple comparison).

$\S$ Significant interaction between age group and trimester: $P<0.05$ (repeated-measures two-factor ANOVA).

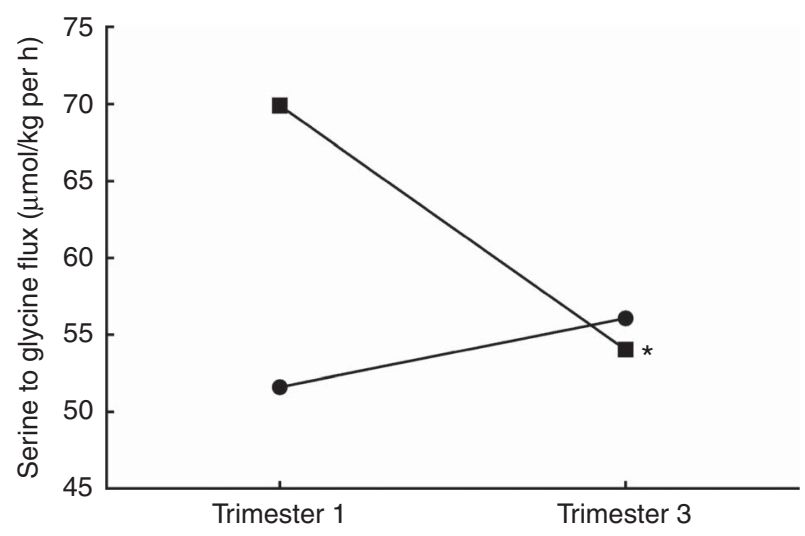

Fig. 1. Weight-specific glycine flux derived from serine in pregnant adult women ( $n 10,--)$ and adolescent girls $(n 11,--)$ ) at 13 and 29 weeks of gestation. * Significant interaction between age group and time of pregnancy $(P<0.05)$, as flux decreased significantly from trimester 1 to 3 in the adolescent group (repeated-measures two-factor ANOVA).

length. It is therefore possible that inability of the adolescent girl to maintain glycine synthesis makes her fetus vulnerable to impaired cartilage synthesis, and thus linear growth.

In addition, some studies in pregnant ewes have reported that there is an absence of serine transport from maternal circulation to the fetus ${ }^{(12-14)}$. Maternal serine is used within the utero-placental tissues to synthesise glycine, some of which is delivered into the fetal circulation and used by the fetus to resynthesise serine ${ }^{(14)}$. In human studies, a higher concentration of serine and glycine was observed in the fetal umbilical vein than in a simultaneously obtained maternal arterial sample at term gestation, suggesting increased de novo synthesis by uteroplacental tissues $^{(15,16)}$. In our present study, the percentage of serine flux derived from glycine (3-5\%) was much less compared with the percentage of glycine flux derived from serine (37-42\%), suggesting that maternal glycine supply was paramount and was being sustained to a large extent by de novo synthesis from serine. Therefore, a contributing factor to the slower glycine flux in adolescent girls in late pregnancy is
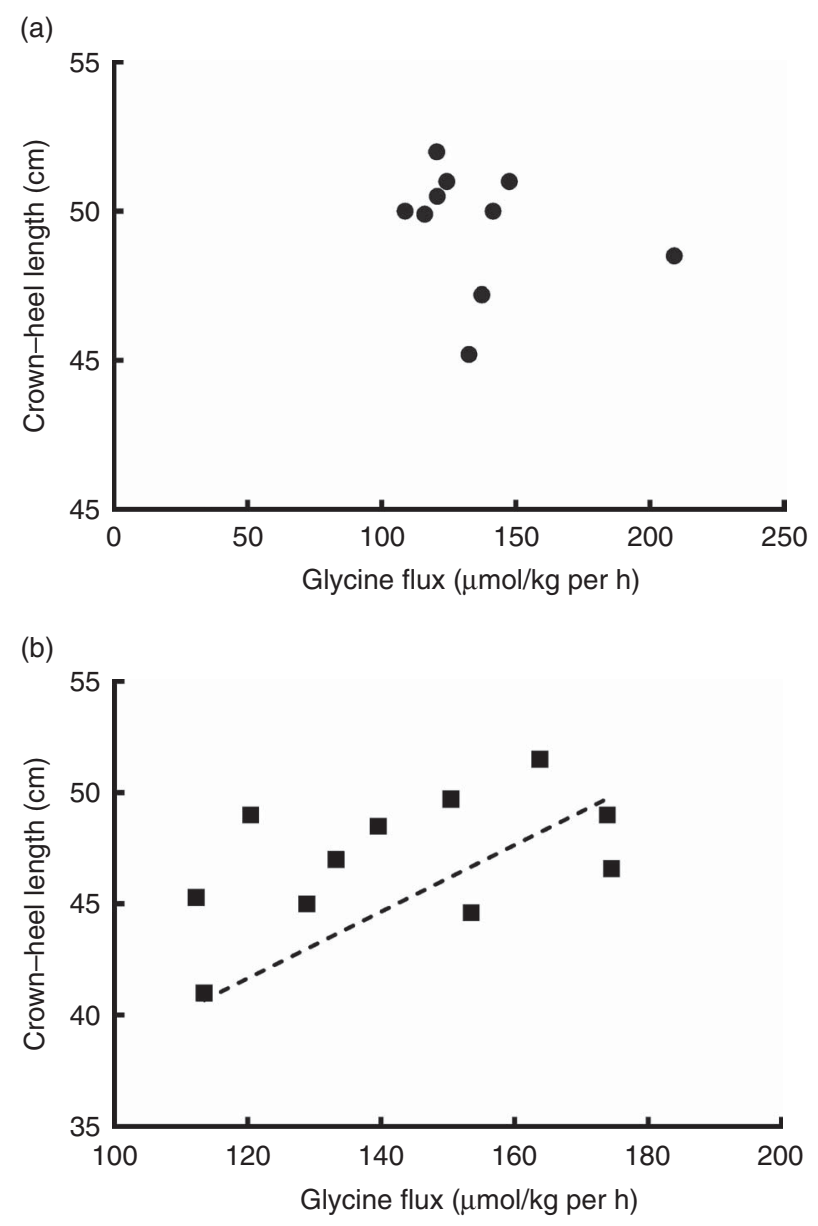

Fig. 2. Association between newborn length and third trimester (29 weeks of pregnancy) glycine flux in pregnant adult women (a) and adolescent girls (b). (a) Pearson's $r-0.26, P=0.24$; (b) Pearson's $r 0.52, P=0.05$.

decreased synthesis from serine. This is supported by the $23 \%$ reduction in serine-derived glycine from the first to the third trimester in the pregnant adolescents. 
An obvious interpretation of these findings is that there is an overall shortage in the supply of serine to support glycine synthesis. In the present study, although serine flux tended to be lower by $17 \%$ in trimester 3 in the adolescent group, the change was not statistically significant. Serine flux in the adult group in trimester 3 was not different from trimester 1 , but it was slightly lower (approximately 6\%). However, in a study by Kalhan et al. ${ }^{(17)}$, they reported that serine flux in adult women was significantly lower in late pregnancy compared with early pregnancy. The authors suggested that the lower serine kinetics in late pregnancy may be part of a general down-regulation of $\alpha$-amino $\mathrm{N}$ turnover related to a decreased rate of branchedchain amino acid transamination to facilitate $\mathrm{N}$ conservation and accretion. In agreement, in an earlier report of data from this project $^{(11)}$, urea flux, an index of protein and amino acid catabolism, decreased from the first to the third trimester in both pregnant adolescent girls and adult women, indicating that they were conserving more $\mathrm{N}$ in the third trimester. However, leucine oxidation in the third trimester was similar to the first trimester, which does not support a decrease in branched-chain amino acid transamination in late pregnancy. Therefore, the lower serine kinetics in late pregnancy was not because of down-regulation of $\alpha$-amino $\mathrm{N}$ turnover in branched-chain amino acid transamination as suggested by Kalhan et al. ${ }^{(17)}$.

In summary, pregnant adolescents cannot maintain glycine flux in late pregnancy compared with early pregnancy. One factor contributing to the slower glycine flux is decreased synthesis from serine. It is possible that inability of the adolescent girl to maintain glycine synthesis makes her fetus vulnerable to impaired cartilage synthesis, and thus linear growth.

\section{Acknowledgements}

The authors are grateful to the nursing staff of the Obstetrics ward at the University Hospital of the West Indies for their care of the subjects.

This research was supported with federal funds from the US Department of Agriculture, Agricultural Research Service under Cooperative Agreement Number 58-6250-6001 and funds from the International Atomic Energy Agency.

All the authors contributed to different aspects of the study, including the design of the study, data collection, sample analysis, data interpretation and writing of the manuscript as follows: F. J., M. M. T. and A. A. J. designed and supervised various aspects of the study; M. M. T., R. G. and T. M. B. recruited the participants, conducted the experiments, processed samples and took care of the subjects; J. W. H., G. J. T. and S. K. C analysed samples and calculated data; and M. M. T., A. A. J., F. J. and J. W. H. analysed and interpreted the data and wrote the manuscript.

None of the authors has any conflicts of interest with the funding agencies.

\section{References}

1. Fraser AM, Brockert JE \& Ward RH (1995) Association of young maternal age with adverse reproductive outcomes. $N$ Engl J Med 332, 1113-1117.

2. Jackson J, Eggleston E, Lee A, et al. (1997) Reproductive knowledge, attitudes and behaviour among young addlescents in Jamaica. Soc Econ Stud 46, 95-109.

3. Duggleby SL \& Jackson AA (2001) Relationship of maternal protein turnover and lean body mass during pregnancy and birth length. Clin Sci (Lond) 101, 65-72.

4. Kalhan S, Rossi K, Gruca L, et al. (1997) Glucose turnover and gluconeogenesis in human pregnancy. J Clin Invest 100, $1775-1781$

5. Lemons JA, Adcock EW 3rd, Jones MD Jr, et al. (1976) Umbilical uptake of amino acids in the unstressed fetal lamb. J Clin Invest 58, 1428-1434.

6. Lamers Y, Williamson J, Gilbert LR, et al. (2007) Glycine turnover and decarboxylation rate quantified in healthy men and women using primed, constant infusions of [1,2-(13)C2] glycine and [(2)H3]leucine. J Nutr 137, 2647-2652.

7. Lamers Y, Williamson J, Theriaque DW, et al. (2009) Production of 1-carbon units from glycine is extensive in healthy men and women. J Nutr 139, 666-671.

8. Meier P, Teng C, Battaglia FC, et al. (1981) The rate of amino acid nitrogen and total nitrogen accumulation in the fetal lamb. Proc Soc Exp Biol Med 167, 463-468.

9. Thame M, Fletcher H, Baker T, et al. (2010) Comparing the in vivo glycine fluxes of adolescent girls and adult women during early and late pregnancy. Br J Nutr 104, 498-502.

10. Gregory JF 3rd, Cuskelly GJ, Shane B, et al. (2000) Primed, constant infusion with [2H3]serine allows in vivo kinetic measurement of serine turnover, homocysteine remethylation, and transsulfuration processes in human one-carbon metabolism. Am J Clin Nutr 72, 1535-1541.

11. Thame MM, Hsu JW, Gibson R, et al. (2014) Adaptation of in vivo amino acid kinetics facilitates increased amino acid availability for fetal growth in adolescent and adult pregnancies alike. Br J Nutr 112, 1779-1786.

12. Chung M, Teng C, Timmerman M, et al. (1998) Production and utilization of amino acids by ovine placenta in vivo. Am J Physiol 274, E13-E22.

13. Geddie G, Moores R, Meschia G, et al. (1996) Comparison of leucine, serine and glycine transport across the ovine placenta. Placenta 17, 619-627.

14. Moores RR Jr, Rietberg CC, Battaglia FC, et al. (1993) Metabolism and transport of maternal serine by the ovine placenta: glycine production and absence of serine transport into the fetus. Pediatr Res 33, 590-594.

15. Cetin I, Hirst K, Corbetta C, et al. (1991) Plasma and erythrocyte amino acids in mother and fetus. Biol Neonate 60, 83-91.

16. Hayashi S, Sanada K, Sagawa N, et al. (1978) Umbilical veinartery differences of plasma amino acids in the last trimester of human pregnancy. Biol Neonate 34, 11-18.

17. Kalhan SC, Gruca LL, Parimi PS, et al. (2003) Serine metabolism in human pregnancy. Am J Physiol Endocrinol Metab 284, E733-E740. 